\title{
Self-Consistent Theoretical Models of Collapsing Pre-Stellar Cores
}

\author{
Valery I. Shematovich ${ }^{1}$, Boris M. Shustov ${ }^{1}$, Dmitri S. Wiebe ${ }^{1}$, \\ Yaroslav N. Pavlyuchenkov ${ }^{1}$, and Zhi-Yun $\mathbf{L i}^{2}$ \\ ${ }^{1}$ Institute of Astronomy of the Russian Academy of Sciences, 48 Pyatnitskaya str., \\ 119017 Moscow, Russia \\ email: shematov@inasan.rssi.ru \\ ${ }^{2}$ Department of Astronomy, University of Virginia, P.O. Box 3818, Charlottesville, \\ VA 22903, USA \\ email: zl4h@virginia.edu
}

\begin{abstract}
We present a coupled dynamical and chemical model for collapsing pre-stellar cores (Li et al. 2002; Shematovich et al. 2003a,b; Pavlyuchenkov et al. 2003). It treats the dynamics of thermally and magnetically supported cores in 1D, with an extended chemical network incorporated. The latest version of the model includes UV-irradiation of the core envelope. We have also developed a 2D Monte Carlo model of radiative transfer to compute molecular line profiles for comparison with observations.

The model allowed us to constrain evolutionary scenarios for collapsing pre-stellar cores, to calculate molecular line profiles from the spatial distribution of chemical species and the velocity field, and to characterize the chemical properties of dense cores.

We have determined line profiles along multiple lines of sight through a given pre-stellar core. This allowed us to compare model predictions with the observational maps of molecular lines available for L1544 and other well studied cores. The comparison of synthetic and observed line profile maps contributed to the understanding of the velocity field and pattern of chemical differentiation observed in individual cores.
\end{abstract}

Keywords. line: profiles — ISM: magnetic fields — ISM: molecules — stars: formation — ISM: individual (L1544)

\section{Introduction}

It is by now well known that the chemical composition of starless (pre-stellar) cores is highly inhomogeneous (e.g., M. Tafalla, this volume). Some (like N-bearing) molecules tend to be centrally peaked, while distributions of the others (like CO or S-bearing species) are reminiscent of concentric shells forming a so-called onion-like structure. One way to study this inhomogeneity is to fit the observed line profiles with theoretical ones computed using some ad hoc representation for the radial abundance profile.

The simplest function that mimics the behavior of molecular abundances in starless cores is a step function in which two abundance values are adopted. One of them is attributed to the core envelope, while the other is associated with the dense central region to take molecular freeze-out into account. Smoother representations are also possible, such as a power law or an exponential function. Observations are used to find the "best fit" parameters that provide the closest agreement between theoretical and observed abundances, column densities, line profiles, etc. This approach is utilized by Tafalla et al. (2002), Lee et al. (2003), Jørgensen et al. (2004), Doty et al. (2004), as well as in other studies. 
Table 1. Timescales in pre-stellar cores

\begin{tabular}{|c|c|c|c|}
\hline Process & Mechanism & Timescale (yr) & $\begin{array}{l}\text { Timescale for } \\
n=10^{4} \mathrm{~cm}^{-3}\end{array}$ \\
\hline Chemistry & Cosmic-ray ionization $^{a, b}$ & $2.6 \times 10^{9} / n$ & $2.6 \times 10^{5}$ \\
\hline Freeze-out & Gas-grain collision & $3 \times 10^{9} / n$ & $3 \times 10^{5}$ \\
\hline Cooling & Radiative emissions & $10^{6}$ & $10^{6}$ \\
\hline Collapse & Gravity & $2.4 \times 10^{7} / \sqrt{n}$ & $2.4 \times 10^{5}$ \\
\hline $\begin{array}{l}\text { Ambipolar } \\
\text { diffusion }\end{array}$ & Ion-neutral drift ${ }^{c}$ & $4 \times 10^{13} x_{e}$ & $4 \times 10^{5}$ \\
\hline
\end{tabular}

${ }^{a}$ assuming cosmic ray ionization rate $\zeta=10^{-17} \mathrm{~s}^{-1}$;

${ }^{b} n$ is the number density of $\mathrm{H}$ nuclei;

${ }^{c} x_{e}$ is the fractional ionization.

Lee et al. (2005) have shown that the simplified representations with "best fit" parameters do reproduce the general abundance trends both in pre-stellar cores and at later stages, when a central heating source has already appeared. However, this technique is only able to shed some light on the current state of an object without giving any clues to its past evolution. The real way to understand the initial conditions of star formation and to elucidate the details of pre-stellar collapse is presented by dynamical models.

The simultaneous modeling of dynamical and chemical evolution is a complicated and computationally demanding task. Possible methods to simplify this task include either a simple chemistry on top of complex dynamics (e.g., Nelson \& Langer 1999; Desch \& Mouschovias 2001), or a complicated chemistry on top of simple dynamics. In the latter case the dynamical evolution of the core can be represented by a pre-defined dependence $n(r, t)$ (like a sequence of Bonnor-Ebert spheres), by a known dynamical solution (freefall, Shu or Larson-Penston solution), or by the solution of Euler's equations in 1D. This approach is utilized by Rawlings et al. (1992), Bergin \& Langer (1997), Aikawa et al. (2001, 2003, 2005), Lee et al. (2004) and by others. The important feature of this approach is that the dynamics is modeled separately and, thus, is decoupled from the chemistry.

Why does one care at all about simultaneous modeling of chemistry and dynamics? The reason is that molecular species are not just passive tracers of dynamical evolution. They influence the dynamics via two primary aspects: radiative cooling and ambipolar diffusion. The latter depends on the atomic and molecular ion density and allows the core to contract despite magnetic support. Timescales for various chemical and dynamical processes are summarized in Table 1. For a typical density in molecular clouds all these timescales are comparable. This implies that in models of early stages of pre-stellar evolution, chemistry and dynamics are to be modeled self-consistently, with dynamical parameters (density, temperature) entering the chemical equations and chemistry-dependent parameters (cooling function, ion-neutral drift efficiency) entering the dynamical equations.

The first attempt to account for the chemistry-dependent thermal balance in a hydrodynamical model was made by Gerola \& Glassgold (1978). For a number of years, the self-consistent approach to modeling of starless cores has been developed by the Moscow astrochemistry group. In one series of models, we studied the chemical and dynamical evolution of a UV irradiated core - an evaporated gaseous globule (EGG, see Figure 1). In such models, the equations of hydrodynamics, chemical kinetics, and energy budget are integrated simultaneously, without imposing any critical pre-defined conditions (Shematovich et al. 1997). It has been shown that even a core immersed in the 

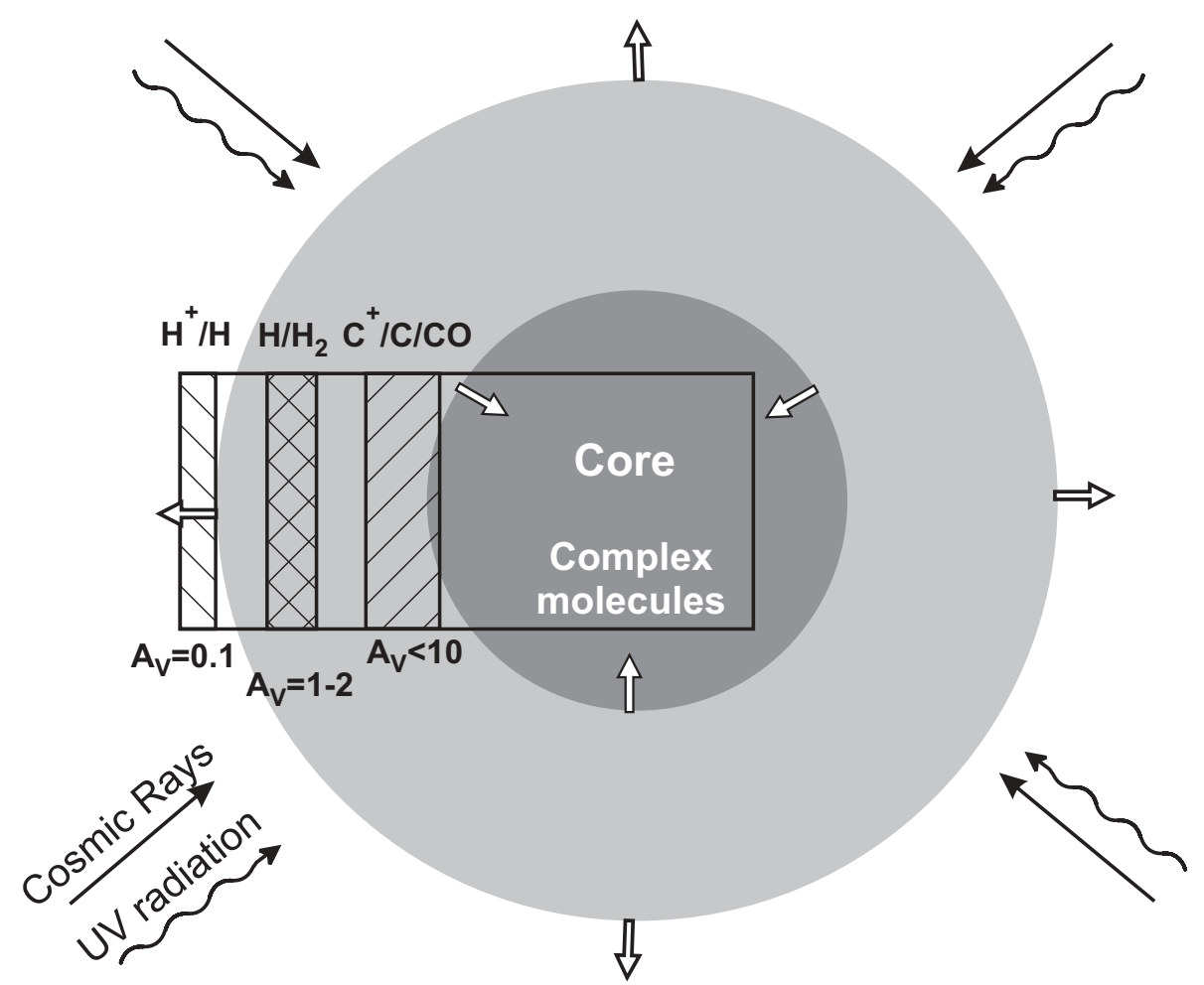

Figure 1. Sketch of a dense pre-stellar core.

diffuse interstellar UV field is separated into two dynamically distinct regions: a collapsing inner part and an expanding envelope. The relative mass fractions in these regions depend on the UV intensity and the initial cloud mass. For low-mass cores $\left(<10 M_{\odot}\right)$ the photoevaporation time is short, and such cores are completely destroyed by diffuse UV radiation. More massive cores can survive weak external heating caused by absorbed UV photons. The core is subject to radiation-driven implosion which is much faster than the "normal" gravitational collapse. On the other hand, heating of the external layers and their evaporation cause the gradual decrease of the core mass. Between the collapsing core and its evaporating envelope a transition region is formed, which propagates inside the core, where the cold dark chemistry has dominated before. Icy mantles evaporate in this transition region, causing local enhancement of some species' abundances, especially, of water vapor.

In another series of models we investigated the contraction of a magnetized core driven by ambipolar diffusion (Li et al. 2002; Shematovich et al. 2003a,b). In this contribution, we present new results related to this model.

\section{Model of a Magnetized Core}

Our model is based on a $1 \mathrm{D}$ representation of the magnetic field, in which the magnetic pressure is retained in the model, while the magnetic tension is neglected. This model is by no means intended to reproduce the kinematic structure of a contracting core; in fact, in Pavlyuchenkov et al. (2003) we show that it does not. However, it does capture the main temporal features of a magnetized core evolution-namely, the quasi-static contraction at the stage when the magnetic support is important and a nearly free-fall collapse afterwards. 
We assume that the core is isothermal at $10 \mathrm{~K}$ and consists of gas and dust that are well mixed. The equations that describe the evolution of a magnetized, self-gravitating cloud are ( $\mathrm{Li} 1999)$

$$
\begin{gathered}
\frac{\partial \rho}{\partial t}=-\frac{1}{r^{2}} \frac{\partial}{\partial r}\left(r^{2} \rho v\right) \\
\frac{\partial v}{\partial t}=-v \frac{\partial v}{\partial r}-\frac{G M_{r}}{r^{2}}-\frac{1}{\rho} \frac{\partial}{\partial r}\left(\rho a^{2}+\frac{B^{2}}{8 \pi}\right) \\
v_{\mathrm{i}}-v=-\tau_{\mathrm{ff}} \nu_{\mathrm{ff}} \frac{1.4}{4 \pi \rho} \frac{\partial}{\partial r}\left(\frac{B^{2}}{2}\right) \\
\frac{\partial B}{\partial t}=-\frac{1}{r} \frac{\partial}{\partial t}\left(r v_{\mathrm{i}} B\right) .
\end{gathered}
$$

Here $a$ is the isothermal sound speed, $M_{r}$ is the mass enclosed within a radius $r, v$ and $v_{\mathrm{i}}$ are velocities of neutral and ionized species, $\tau_{\mathrm{ff}}$ is the free-fall time for density $\rho$, and $\nu_{\mathrm{ff}}$ is the magnetic coupling parameter. The latter value is the ratio of the local free-fall time to the magnetic field-neutral coupling timescale. It is this parameter that defines the connection between the chemical and dynamical evolution of the core. The ion density that is needed to compute $\nu_{\mathrm{ff}}$ is evaluated with a time-dependent chemical model that includes approximately 2000 gas-phase reactions, involving 59 neutral and 83 ionized species from the UMIST 95 ratefile (Millar et al. 1997), as well as 53 grainsurface diffusive reactions (Hasegawa \& Herbst 1993). Gas and dust phases are coupled through adsorption-desorption processes. Desorption energies are taken from Hasegawa \& Herbst (1993) with updates from Aikawa et al. (2001). Adsorption is parameterized with the sticking probability $s$. The core mass is assumed to be $\sim 20 M_{\odot}$, with an initial density of $n_{\mathrm{H}_{2}}=10^{3} \mathrm{~cm}^{-3}$. The initial state of the core is magnetostatic. If there were no ambipolar diffusion, the core would stay in this state indefinitely long.

\section{Chemical Differentiation in the Magnetized Pre-Stellar Cores}

Slow contraction is naturally achieved in a strongly magnetized core, whose evolution is driven by ambipolar diffusion over several dynamic times (Li 1999; Ciolek \& Basu 2000). The relatively long evolution time should leave a strong imprint on the core chemistry, since the chemical and dynamical timescales are comparable. In a magnetic cloud, the interplay between dynamics and chemistry is highly nonlinear: the density evolution affects the chemistry, which determines the abundances of charged particles, which in turn controls the rate of ambipolar diffusion that regulates the density evolution.

The coupled dynamical and chemical model was used to study the chemical appearance of pre-stellar cores (Shematovich et al. 2003a,b) with special attention to the effects of the strength of magnetic fields, initial chemical composition, sticking probability, adsorption energies, cosmic ray ionization rate, and cloud mass. The model results were compared with the data available on the well studied core L1544. The main conclusion from our previous studies is that the model, in which the cloud is magnetically supported for several million years before collapsing dynamically, provides a good overall fit to the observed column densities of various species in L1544. The fit is significantly worse for the non-magnetic model, in which the cloud collapses promptly. In particular, the radius of the ring in the distribution of sulfur-bearing molecules is much smaller than observed. Varying the model parameters, we have been able to build a model which reproduces observed column densities of $\mathrm{CO}, \mathrm{CS}, \mathrm{CCS}, \mathrm{HCO}^{+}, \mathrm{NH}_{3}$, and $\mathrm{N}_{2} \mathrm{H}^{+}$within a factor of 3 . 

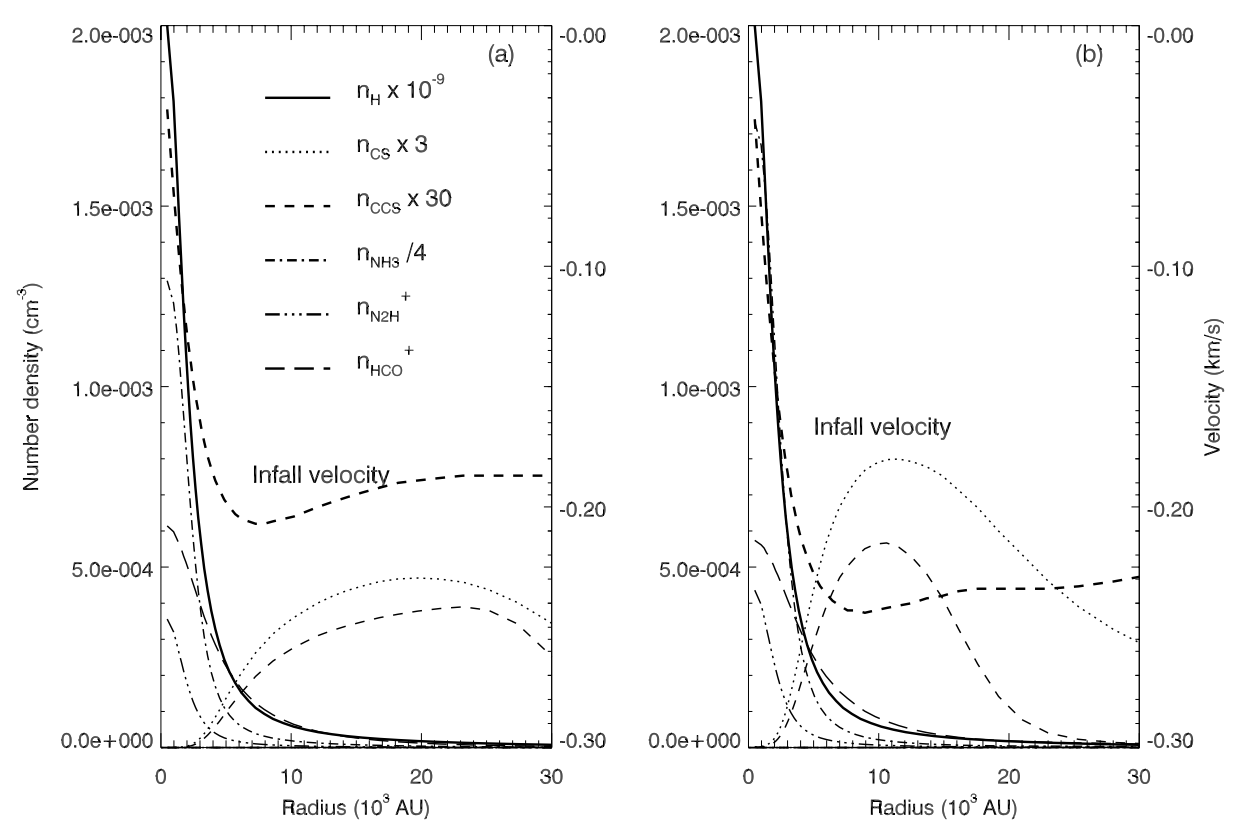

Figure 2. Radial profiles of the volume densities of the hydrogen nuclei (heavy solid line) and 5 selected molecular species, plotted together with the profile of the infall velocity (heavy dashed line) at a time when the $\mathrm{H}_{2}$ central density reaches a value of $10^{6} \mathrm{~cm}^{-3}$. Note that the high-density central region has a relatively small infall speed, and is well traced by the species $\mathrm{NH}_{3}$ and $\mathrm{N}_{2} \mathrm{H}^{+}$, and to a lesser extent, by $\mathrm{HCO}^{+}$. The faster contracting inner envelope is, on the other hand, better traced by the centrally-depleted species CS and CCS. Some curves are shifted by various factors for clarity. (a) Standard model; (b) Same model but with ion sticking probability $s_{i}=1$.

Our previous studies represent an important illustration of the influence that chemistry exerts on dynamics. Cosmic rays are the only noticeable source of ionization in these cores; thus, the fractional ionization is determined by the molecular chemistry, driven by cosmic rays. Varying only parameters of the chemical model we change the contraction timescale by a factor of 3 . In the standard model the density grows from $10^{3}$ to $10^{6} \mathrm{~cm}^{-3}$ over 6.5 Myr. If we raise the sticking probability for ions from 0.3 to 1 , the contraction accelerates due to the decreased abundance of ions, and the evolutionary time shortens to $4.9 \mathrm{Myr}$. If the surface chemistry is ignored in the model, the contraction lasts for 10.9 Myr. Finally, if ion recombination on grains is prohibited, the timescale increases up to 14.4 Myr.

In this paper, we emphasize the dependence of the developed model on the chemical exchange between the gas and dust phases. The standard chemical and dynamical model of the magnetized pre-stellar core of Shematovich et al. (2003a), having sticking probabilities for neutrals $\left(s_{\mathrm{n}}\right)$ and ions $\left(s_{\mathrm{i}}\right)$ equal to 0.3 for both neutrals and ions is analyzed together with the model in which $s_{\mathrm{n}}=0.3$ and $s_{\mathrm{i}}=1$. The higher value of the sticking probability for ions causes a stronger chemical coupling between the gas and dust, and corresponds to the case when dust grains are mainly negatively charged. The chemistry network of our model was updated by taking into account the new data on (i) rate coefficients and branching ratios for dissociative recombination of molecular ions $-\mathrm{H}_{3}^{+}, \mathrm{CH}_{3}^{+}$, $\mathrm{H}_{3} \mathrm{O}^{+}, \mathrm{N}_{2} \mathrm{H}^{+}, \mathrm{NH}_{4}^{+}, \mathrm{CH}^{+}-\mathrm{CH}_{4}^{+}$, etc. (see discussion by Herbst 2005); and (ii) new and re-estimated rate coefficients of neutral-neutral reactions (Smith et al. 2004). 

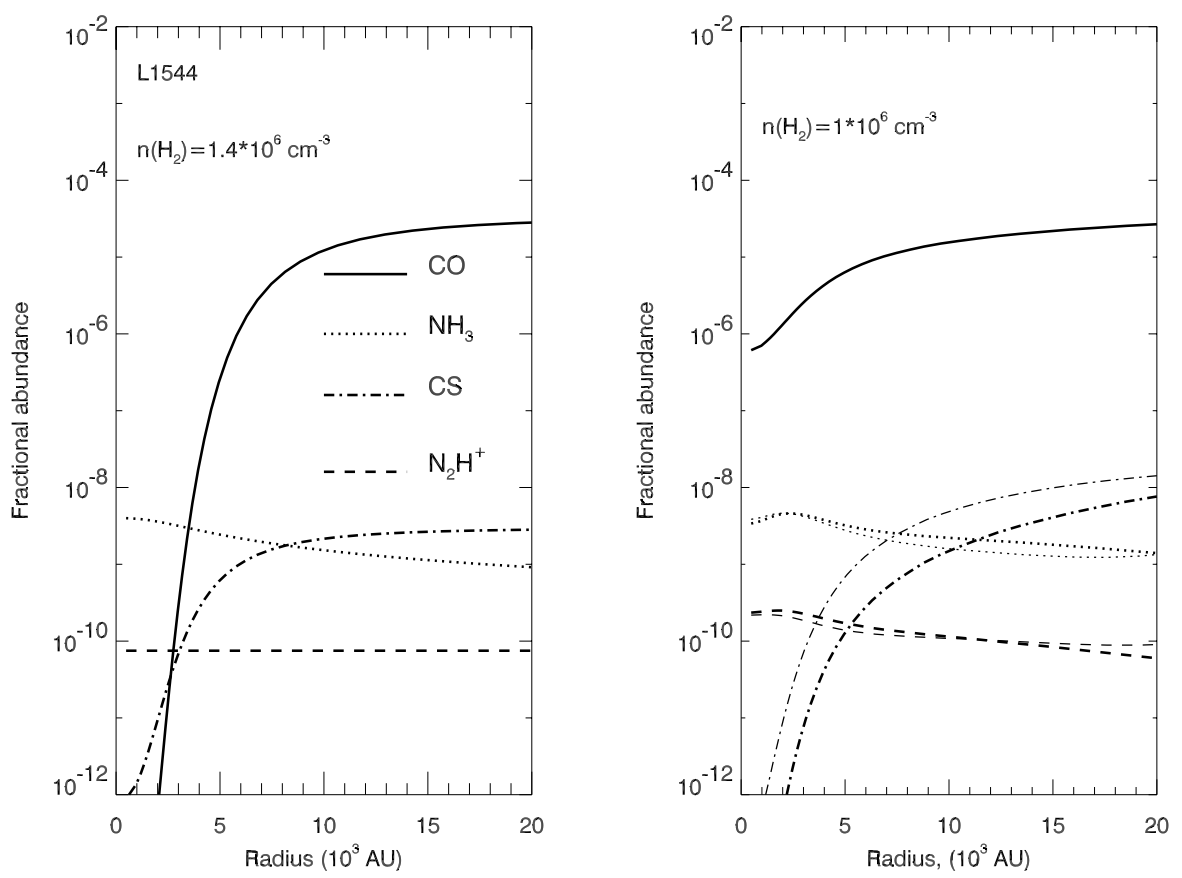

Figure 3. Right: Patterns of chemical differentiation in the core models with ion sticking coefficients $s_{i}=0.3$ (thick lines) and 1 (thin lines). Left: "Best fit" model of line observations of the pre-stellar core L1544 (Tafalla et al. 2002).

Predictions of our standard model on chemistry are in agreement with observations (Tafalla et al. 2002; Tafalla, this volume). As can be seen from Figure 2, the following features are typical of pre-stellar cores approaching a central density of $10^{5}-10^{6} \mathrm{~cm}^{3}$ of hydrogen nuclei:

- There is a general trend for the fractional abundance to decrease toward the core center, with a few exceptions. This central depletion is particularly prominent for the sulphur-bearing molecules CS and CCS.

- The nitrogen-bearing species, such as $\mathrm{NH}_{3}, \mathrm{HCN}$ and $\mathrm{N}_{2} \mathrm{H}^{+}$, show little depletion or even enhancement in the central region. Molecular nitrogen becomes one of the most abundant species in the gas phase.

- CO remains abundant in the outer envelope, but decreases by more than an order of magnitude in the very central region.

- The gas-phase water is strongly depleted, therefore it becomes a main constituent of the icy mantles of dust grains.

Our main conclusion is that the observed chemical differentiation can be explained as a natural consequence of the core evolution. The dense cores, formed from a more diffuse background (strongly magnetized or not) by gravitational contraction, will have a plateau-envelope density structure, and a distinctive velocity field: zero velocity at the center, nearly linear increase in the central plateau region, with a peak somewhere in the inner envelope. If the abundances of $\mathrm{NH}_{3}$ and $\mathrm{N}_{2} \mathrm{H}^{+}$are not depleted or even enhanced, they should preferentially trace the low-speed plateau region. Strongly depleted species, such as CS and CCS, on the other hand, should be more sensitive to the inner envelope, which has a faster infall motion (see Figure 2). The depletion pattern is not 
strongly changed when the sticking probabilities are high, $s_{i}=1$. Nevertheless, in this case we have both higher abundances of sulphur-bearing molecules and higher infall velocity (Figure 2b). The chemical differentiation offers an exciting possibility of determining this distinctive velocity signature, and may directly probe the core growth.

For the set of models considered in Shematovich et al. (2003a,b), we find that the magnetic model with mixed initial metal abundances best reproduces the data on the velocity field and column densities of various species in L1544. Tafalla et al. (2002) modeled the data from L1544 carefully, and deduced fractional abundances for $\mathrm{C}^{18} \mathrm{O}, \mathrm{CS}, \mathrm{N}_{2} \mathrm{H}^{+}$, and $\mathrm{NH}_{3}$, as well as the hydrogen number density as functions of the radius from the center. A comparison between the patterns of chemical differentiation in the core models with ion sticking coefficients $s_{i}=0.3$ (thick lines) and 1 (thin lines) and the "best fit" model of line observations of the pre-stellar core L1544 (Tafalla et al. 2002) is presented in Figure 3 .

Radial distributions of $\mathrm{C}^{18} \mathrm{O}, \mathrm{CS}, \mathrm{N}_{2} \mathrm{H}^{+}$, and $\mathrm{NH}_{3}$ provide a more stringent test of model predictions than the integrated column densities used previously. From this comparison we would like to mention, that the density profile provided by the considered self-consistent models is close to that inferred by Tafalla et al. (2002). Next, the model appears to fit the inferred abundance distributions of all four species reasonably well, particularly for $\mathrm{NH}_{3}$. The most notable discrepancy lies in $\mathrm{C}^{18} \mathrm{O}$ at the smallest radii (inside about $10^{4} \mathrm{AU}$ ), although we note that the distribution in the innermost region is not well constrained observationally because of finite spatial resolution. The predicted $\mathrm{N}_{2} \mathrm{H}^{+}$abundance increases gradually inward, which is different from the inferred constant distribution. The difference is relatively modest, however, within a factor of 2 or so. The predicted CS abundance is significantly higher than the inferred value at large radii (beyond a few times $10^{4} \mathrm{AU}$ ), which may require a better treatment of the sulphur chemistry, which is somewhat uncertain.

\section{Synthetic Line Profiles}

The potential of the coupled dynamical and chemical model is fully realized by constructing and analyzing the synthetic line profiles at various core locations, because such model provides a simultaneous determination of the spatial distribution of a given chemical species and the velocity field, the two ingredients for line profile modeling. Line profiles of species such as $\mathrm{CS}$ and $\mathrm{HCO}^{+}$are routinely employed to deduce infall motions in dense cores. The fact that these species may be depleted poses a problem. Rawlings \& Yates (2001) examined the line profiles in a collapsing core in some detail, and concluded that models assuming chemical uniformity or simple monotonic variations are unacceptable.

We have calculated the line profiles of $\mathrm{C}^{18} \mathrm{O}(1-0)$, CS $(2-1)$, and $\mathrm{HCO}^{+}(3-2)$ transitions for the models with ion sticking coefficient equal to 0.3 (solid lines) and 1 (dashed lines) with the radiative trasfer code URANIA (Pavlyuchenkov et al. 2003; Pavlyuchenkov \& Shustov 2004), and have compared these spectra with observed line profiles for L1544 taken from Tafalla et al. (2002), Lee et al. (2001), and Lee et al. (2003), correspondingly. This comparison is shown in Figure 4. We see that the intensities of the calculated line profiles are slightly higher than the observed values and the lines are double-peaked with strong dips for $\mathrm{CS}(2-1)$, and $\mathrm{HCO}^{+}(3-2)$ lines. These features are mainly caused by the relatively high infall speed in the model core and excess abundances in the outer envelope of the pre-stellar core. The synthetic line profiles calculated with the high ion sticking probability are shown by dashed lines. These profiles have a slightly higher intensity because of the shorter time for molecules to freeze out. The calculated $\mathrm{C}^{18} \mathrm{O}$ (1-0) line profile is still double-peaked compared with the observed line because of the 

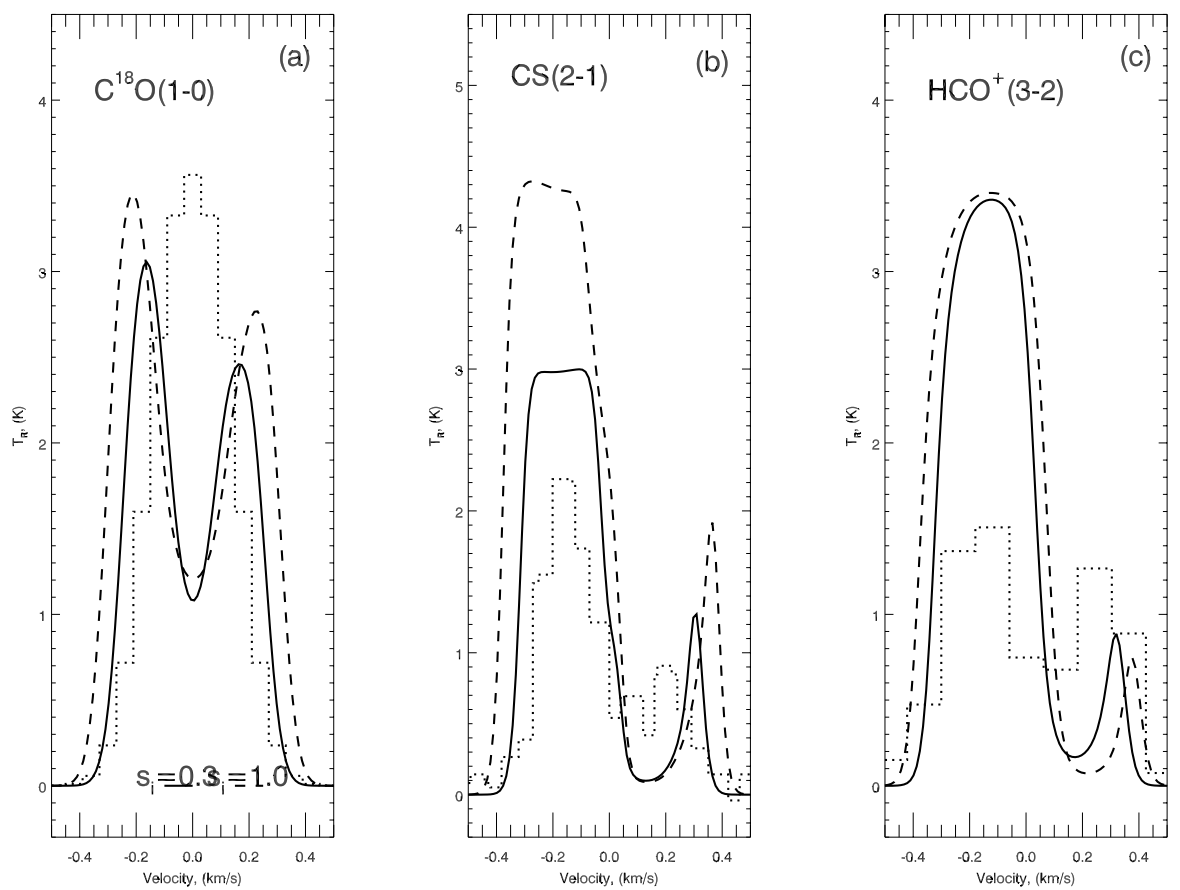

Figure 4. Comparison between observed and synthetic line profiles of $\mathrm{C}^{18} \mathrm{O}(1-0), \mathrm{CS}(2-1)$, and $\mathrm{HCO}^{+}(3-2)$ transitions for models with ion sticking coefficients $s_{i}=0.3$ (solid lines) and 1 (dashed lines). Observed molecular lines from the L1544 core are shown by dotted lines. (a) $\mathrm{C}^{18} \mathrm{O}(1-0)$ line profile was taken from Tafalla et al. (2002); (b) $\mathrm{CS}(2-1)$ line profile was taken from Lee et al. (2002); (c) $\mathrm{HCO}^{+}(3-2)$ line profile was taken from Lee et al. (2003).

relatively high $\mathrm{CO}$ abundance in the core envelope. These differences between modeled and observed line profiles are mainly caused by the high infall speeds obtained in the considered models and are a shortcoming of the 1D model of the dynamical and chemical evolution of magnetized starless cores. They can be overcome by reducing the infall speed to the level of half of the sound speed, and by reducing the $\mathrm{CO}$ abundance by exposing the outer core envelope to interstellar UV radiation.

There is an additional indication of the more complex velocity field in the L1544 core. In Figure 5, we compare the calculated and observed maps of the $\mathrm{HCO}^{+}(3-2)$ transitions in this core (Gregersen \& Evans 2000). It is seen that it is impossible to reproduce in the calculated map the changing ratio between blue and red wings in the $\mathrm{HCO}^{+}(3-2)$ line profiles. This effect could be referred to the slow rotation of the L1544 core. Such a complicated velocity field can be hopefully reproduced by $2 \mathrm{D}$ or $3 \mathrm{D}$ versions of the self-consistent chemical and physical model of pre-stellar core evolution.

\section{Conclusions}

Self-consistent chemical and dynamical models seem to be very promising for investigation of the very early stages of star formation. Many features of pre-stellar cores are interpreted in a natural manner. The presented results suggest that the chemical differentiation observed in L1544 can be explained quantitatively in the context of the standard scenario for isolated low-mass core formation, involving ambipolar diffusion over several dynamic times. We plan to strengthen this conclusion by refining our model, and 


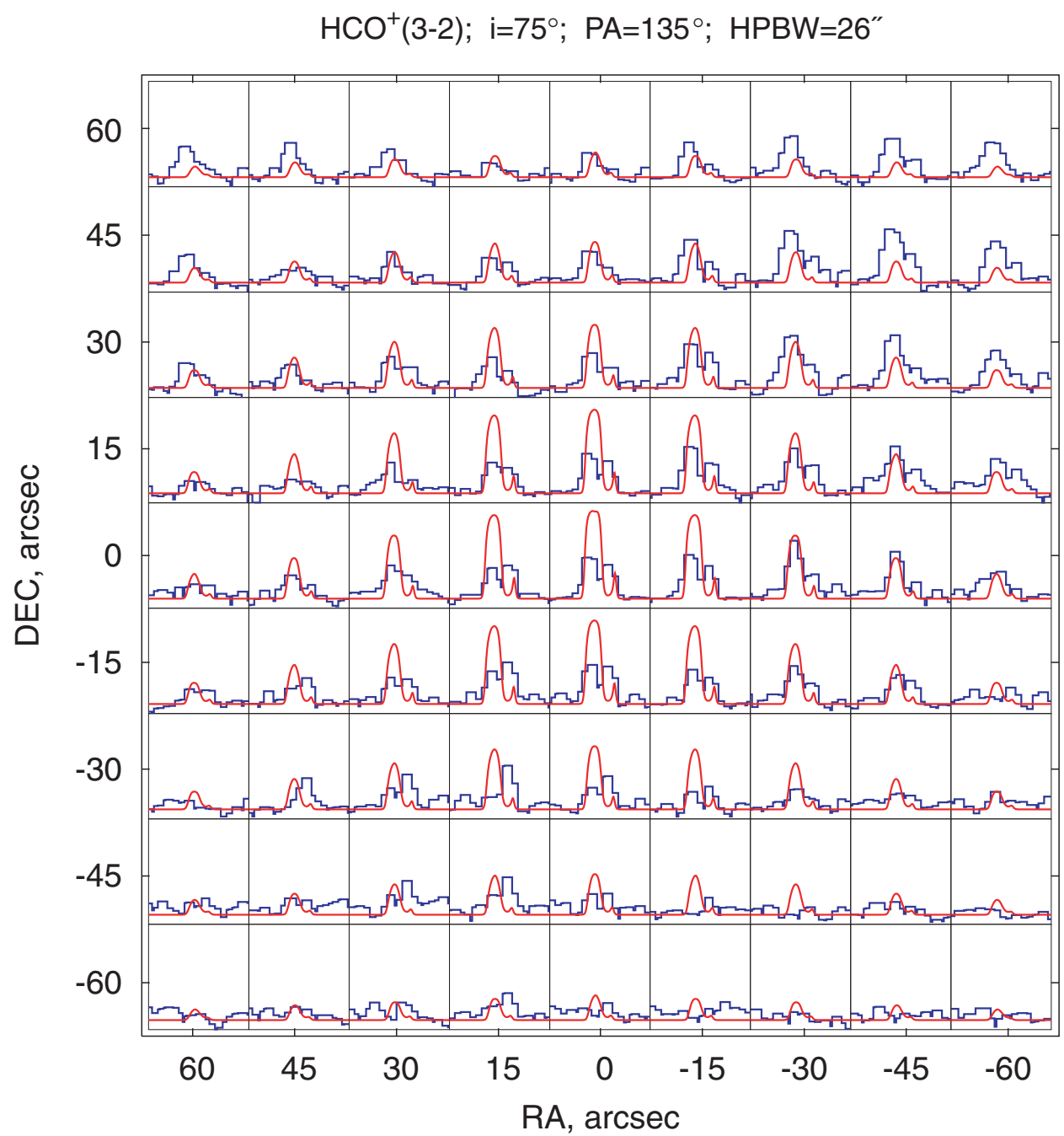

Figure 5. Map of the observed and synthetic line profiles of $\mathrm{HCO}^{+}(3-2)$ transitions for the standard model. Observed $\mathrm{HCO}^{+}(3-2)$ map for the L1544 core, taken from Gregersen \& Evans (2000), is shown by histogram-like curves.

developing $2 \mathrm{D}$ and $3 \mathrm{D}$ versions of our self-consistent chemical and dynamical model needed to improve the interpretation of high-resolution observations.

\section{Acknowledgements}

The Moscow astrochemistry group is supported by RFBR grant number 04-02-16637. Work on star formation at the University of Virginia is supported in part by NSF grant AST-0307368.

\section{References}

Aikawa, Y., Herbst, E., Roberts, H., \& Caselli, P. 2005, Ap. J. 620, 330

Aikawa, Y., Ohashi, N., \& Herbst, E. 2003, Ap. J. 593, 906

Aikawa, Y., Ohashi, N., Inutsuka, Sh., Herbst, E., \& Takakuwa, Sh. 2001, Ap. J. 552, 639

Bergin, E.A. \& Langer, W.D. 1997, Ap. J. 486, 316 
Ciolek, G.E. \& Basu, S. 2000, Ap. J. 529, 925

Desch, S.J. \& Mouschovias, T.Ch. 2001, Ap. J. 550, 314

Doty, S.D., Schöier, F.L., \& van Dishoeck, E.F. 2004, A\&A 418, 1021

Gerola, H. \& Glassgold, A.E. 1978, Ap. J. Suppl. 37, 1

Gregersen, E.M., \& Evans, N.J. 2000, Ap. J. 538, 260

Hasegawa, T.I., \& Herbst, E. 1993, MNRAS 263, 589

Herbst, E. 2005, J. Phys.: Conf. Ser. 4, 17

Jørgensen, J.K., Schöier, F.L., \& van Dishoeck, E.F. 2004, A\&\&A 416, 603

Lee, C.-W., Myers, P.C., \& Tafalla, M. 2001, Ap. J. Suppl. 136, 703

Lee, J.-E., Bergin, E.A., \& Evans, N.J. 2004, Ap. J. 617, 360

Lee, J.-E., Evans, N.J., \& Bergin, E.A. 2005, Ap. J., in press (astro-ph/0506086)

Lee, J.-E., Evans, N.J., Shirley, Y.L., \& Tatematsu, K. 2003, Ap. J. 583, 789

Li, Z.-Y. 1999, Ap. J., 526, 806

Li, Z.-Y., Shematovich, V.I., Wiebe, D.S., \& Shustov, B.M. 2002, Ap. J. 569, 792

Millar, T.J., Farquhar, P.R.A., \& Willacy, K. 1997, A\& AS 121, 139

Nelson, R.P. \& Langer, W.D. 1999, Ap. J. 524, 923

Pavlyuchenkov, Ya.N. \& Shustov, B.M. 2004, Astronomy Reports 48, 315

Pavlyuchenkov, Ya.N., Shustov, B.M., Shematovich, V.I., Wiebe, D.S., \& Li, Z.-Y. 2003, Astronomy Reports 47, 176

Rawlings, J.M.C. \& Yates, J.A. 2001, MNRAS 326, 1423

Rawlings, J.M.C., Hartquist, T.W., Menten, K.M., \& Williams, D.A. 1999MNRAS 255, 471

Shematovich, V.I., Wiebe, D.S., \& Shustov, B.M. 1997, MNRAS 292, 601

Shematovich, V.I., Wiebe, D.S., Shustov, B.M., \& Li, Z.-Y., 2003a, Ap. J. 588, 894

Shematovich, V.I., Wiebe, D.S., Shustov, B.M., Pavlyuchenkov, Ya.N., \& Li, Z.-Y. 2003b, in Chemistry as a diagnostic of star formation, eds. C.L. Curry \& M. Fich (Ottawa: NRC Research Press), p. 97

Shu, F. 1977, Ap. J. 214, 488

Smith, I.W.M., Herbst, E., \& Chang, Q. 2004, MNRAS 350, 323

Tafalla, M., Myers, P.C., Caselli, P., Walmsley, C.M., \& Comito, C. 2002, Ap. J. 569, 815

\section{Discussion}

AIKAWA: You showed that the model of Shu (1977) (without magnetic field) fit the observed line profiles better than your model with ambipolar diffusion. Usually people expect that a magnetized core collapses more slowly than that without magnetic field. Would you tell me why you obtained a faster infall velocity than Shu's model?

Shematovich: Our new self-similar model is a generalization of Shu-like solution to the pre-stellar phase, including an infall speed that is treated as a free parameter. It is set to a value that is about half of the sound speed for the particular example shown.

VITI: Regarding the problem with $\mathrm{HCO}^{+}(3-2)$ line observations, have you included rotation in your models? Could rotation affect your fits?

Shematovich: Yes, the core rotation can improve the approach of the synthetic map to the observed one. As we are talking about self-consistent models, then the correct inclusion of core rotation into the model can be done at least in the frame of a $2 \mathrm{D}$ model of core dynamics. 